\title{
Seismoelectric interface response: Experimental results and forward model
}

\author{
M. D. Schakel ${ }^{1}$, D. M. J. Smeulders ${ }^{2}$, E. C. Slob ${ }^{1}$, and H. K. J. Heller ${ }^{1}$
}

\begin{abstract}
Understanding the seismoelectric interface response is important for developing seismoelectric field methods for oil exploration and environmental/engineering geophysics. The existing seismoelectric theory has never been validated systematically by controlled experiments. We have designed and developed an experimental setup in which acoustic-toelectromagnetic wave conversions at interfaces are measured. An acoustic source emits a pressure wave that impinges upon a porous sample. The reflected electric-wave potential is recorded by a wire electrode. We have also developed a full-waveform electrokinetic theoretical model based on the Sommerfeld approach and have compared it with measurements at positions
\end{abstract}

perpendicular and parallel to the fluid/porous-medium interface. We performed experiments at several salinities. For $10^{-3}$ and $10^{-2} \mathrm{M}$ sodium chloride $(\mathrm{NaCl})$ solutions, both waveforms and amplitudes agree. For $10^{-4} \mathrm{M} \mathrm{NaCl}$, however, amplitude deviations occur. We found that a single amplitude field scaling factor describes these discrepancies. We also checked the repeatability of experiments. The amplitudes are constant for the duration of an experiment (1-4 hours) but decrease on longer time scales ( $\sim 24$ hours). However, the waveforms and spatial amplitude pattern of the electric wavefield are preserved over time. Our results validate electrokinetic theory for the seismic-to-electromagnetic-wave conversion at interfaces for subsurface exploration purposes.

\section{INTRODUCTION}

When the grain surfaces of soils and rocks are in contact with a fluid electrolyte, they typically acquire a chemically bound surface charge that is balanced by mobile counterions in a thin fluid layer surrounding the grains. The bound charge is immobile, whereas the counterions can move with the fluid. At the interface between the immobile and counterions, the so-called zeta potential is defined. A comprehensive theory for coupled seismic and electromagnetic (EM) wave propagation with the zeta potential as a key parameter is derived by Pride (1994) From research based on this electrokinetic theory, one can distinguish two seismoelectric effects: (1) a coseismic (electric) field is coupled to seismic waves and therefore propagates with seismic wave velocity (e.g., Pride and Haartsen, 1996) and (2) a seismic wave that traverses an interface with a contrast in electrical or mechanical properties produces EM waves that propagate outside the support of the seismic waves with much higher EM wavespeeds (e.g.,
Pride and Haartsen, 1996; Haartsen and Pride, 1997). These effects have been verified experimentally in the laboratory (e.g., Zhu et al., 1999, 2000; Zhu and Toksöz, 2003; Block and Harris, 2006; Bordes et al., 2006) and in the field (e.g., Long and Rivers, 1975; Butler et al., 1996; Mikhailov et al., 1997; Beamish, 1999; Garambois and Dietrich, 2001; Dupuis et al., 2007; Haines et al., 2007). Also, the coseismic magnetic fields associated with a Stoneley wave (Zhu and Toksöz, 2005) and with a shear wave have been measured (Bordes et al., 2006, 2008).

Electrokinetic theory combines poroelastic and EM theory through coupling in flux/force relations. The so-called dynamic electrokinetic coupling coefficient that occurs in the flux/force relations has been validated experimentally (Reppert et al., 2001). Seismoelectric exploration can combine seismic resolution and the sensitivity to pore fluids of EM methods (Haines et al., 2007). Garambois and Dietrich (2001), Block and Harris (2006), and Dupuis and Butler (2006) compare measured and theoretically predicted coseismic electric field amplitudes.

Manuscript received by the Editor 20 July 2010; revised manuscript received 29 March 2011; published online 16 June 2011, corrected version published 23 June 2011

${ }_{1}^{1}$ Delft University of Technology, Department of Geotechnology, Delft, Netherlands. E-mail: m.d.schakel@tudelft.nl; e.c.slob@tudelft.nl; h.k.j.heller@tudelft.nl.

${ }^{2}$ Eindhoven University of Technology, Department of Mechanical Engineering, Eindhoven, Netherlands, and Delft University of Technology, Delft, Netherlands. E-mail: d.m.j.smeulders@tudelft.nl.

(C) 2011 Society of Exploration Geophysicists. All rights reserved. 
The numerically predicted amplitude-decay pattern of the seismoelectric conversion at an interface (e.g., Haartsen and Pride, 1997; Garambois and Dietrich, 2002; Haines and Pride, 2006) has been observed by several workers (e.g., Butler et al., 1996; Mikhailov et al., 1997; Dupuis et al., 2007). Mikhailov et al. (1997) compare field measurements of this pattern as a function of horizontal distance with a numerical model and find reasonable agreement, and Charara et al. (2009) model and measure seismoelectric signals at a fluid/porous-medium interface in a laboratory experiment. However, Charara et al. do not consider the spatial variation of reflected waveform and amplitude.

In this article, we test the predictive power of electrokinetic theory for the seismoelectric interface response. Laboratory measurements and modeling on the basis of the Sommerfeld approach and Pride's electrokinetic theory are presented. Measurements are conducted at several positions in a water tank in which a fluidsaturated porous sample is immersed. Excellent agreement between theory and experiments for waveforms and spatial amplitude pattern is found. Measured amplitudes are shown to be dependent on salinity. Only a single amplitude field scaling factor is necessary to find absolute agreement. Our research validates electrokinetic theory for the seismoelectric interface response.

\section{SEISMOELECTRIC EXPERIMENTAL SETUP}

The experimental setup consists of a $58 \times 39 \times 28 \mathrm{~cm}$ water tank in which a 1.125 inch diameter $\mathrm{P}$-wave Panametrics source transducer (model V3638, 500-kHz center frequency), a $380 \mu \mathrm{m}$ diameter A-M Systems silver/silver chloride $(\mathrm{Ag} / \mathrm{AgCl})$

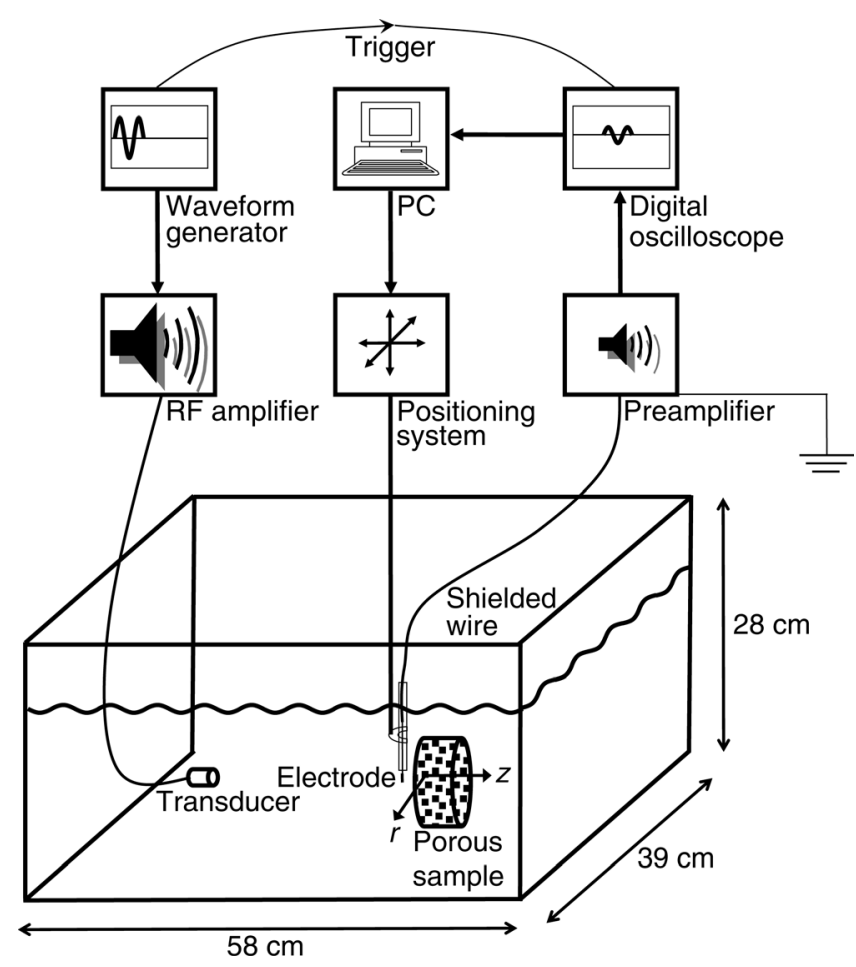

Figure 1. Schematic of the experimental setup. The electrode is clamped in a computer-controlled mounting bracket. Electrode recordings are with respect to ground level. The shielding of the wire electrode is also connected to ground level. The $r$ - and $z$-axes coincide with those of Figure 3. electrode, and a Technoglas porous sample (P3 glass filter) are mounted (see Figure 1). The sample is made of sintered 40-100 $\mu \mathrm{m}$ glass particles that are carefully saturated with and immersed in a $10^{-3} \mathrm{M}$ sodium chloride $(\mathrm{NaCl})$ solution. A 500 $\mathrm{kHz}$ single sine pulse with a $500 \mathrm{mV}$ peak-to-peak amplitude from an Agilent waveform generator (model 33220A) is fed into an ENI amplifier (model 2100L RF Power Amplifier) set at 50 $\mathrm{dB}$ gain. The output signal is coupled into a piezoelectric source transducer. The electrode is clamped in a computer-controlled mounting bracket so it can be positioned in the water layer with high accuracy. An Analogic preamplifier (model D1000) and a Yokogawa oscilloscope (model DL4200) are used for recording. Parameter values are given in Table 1.

The cylindrical coordinate system $(r, z)$ is also indicated in Figure 1. The $z$-axis connects the centers of the sample and the transducer. At several positions along the $z$-axis (Figure 1), electric potential measurements are performed. These on-axis recordings, averaged over 8192 source pulses, are shown in Figure 2a. A $144-896 \mathrm{kHz}$ numerical band-pass filter is applied in all measurements. The amplifier is calibrated with respect to amplitude and waveform using typical time signals. An electric pulse arrives at approximately $0.1 \mathrm{~ms}$ at each position, corresponding to the traveltime of the acoustic wave from the source to the sample surface. Because of the large EM wavespeed (approximately $1.1 \times 10^{7} \mathrm{~m} / \mathrm{s}$ ), the electric pulses arrive almost simultaneously at each position. Note that the pulse decays in

Table 1. Parameters of the porous medium. The values for $\boldsymbol{K}_{s}$, $K_{f}, \eta, \rho_{f}, \Lambda, \epsilon_{f}, \epsilon_{s}$, and $\mu$ are taken from the literature (see below). Values for $K_{b}$ and $\boldsymbol{G}$ are derived from experiments, and $\phi, \rho_{s}, k_{0}$, and $\alpha_{\infty}$ are measured directly. When applicable, fluid parameters are the same as for the porous medium. The temperature is $293.15 \mathrm{~K}$.

\begin{tabular}{|c|c|c|c|}
\hline Symbol & Parameter & Value & Units \\
\hline$K_{s}$ & Bulk modulus skeleton grains ${ }^{\mathrm{a}}$ & $49.9 \times 10^{9}$ & $\mathrm{~Pa}$ \\
\hline$K_{f}$ & Bulk modulus (pore) fluid ${ }^{\mathrm{b}}$ & $2.2 \times 10^{9}$ & $\mathrm{~Pa}$ \\
\hline$\eta$ & Pore fluid viscosity ${ }^{\mathrm{b}}$ & $1 \times 10^{-3}$ & $\mathrm{Pas}$ \\
\hline$\rho_{f}$ & (Pore) fluid density ${ }^{\mathrm{b}}$ & 998 & $\mathrm{~kg} / \mathrm{m}^{3}$ \\
\hline$\Lambda$ & $\begin{array}{l}\text { Weighted pore-volume-to- } \\
\text { surface ratioc }\end{array}$ & $1.229 \times 10^{-5}$ & $\mathrm{~m}$ \\
\hline$\epsilon_{f}$ & $\begin{array}{l}\text { Relative permittivity of the } \\
\text { (pore) fluid }{ }^{\mathrm{b}}\end{array}$ & 80.1 & - \\
\hline$\epsilon_{s}$ & Relative permittivity of the solid ${ }^{\mathrm{b}}$ & 4 & - \\
\hline$\mu$ & $\begin{array}{l}\text { (Fluid) magnetic permeability } \\
\left(=\mu_{0}\right)\end{array}$ & $4 \pi \times 10^{-7}$ & $\mathrm{H} / \mathrm{m}$ \\
\hline$K_{b}$ & $\begin{array}{l}\text { Bulk modulus framework } \\
\text { of grains }\end{array}$ & $6.6 \times 10^{9}$ & $\mathrm{~Pa}$ \\
\hline$G$ & $\begin{array}{l}\text { Shear modulus framework } \\
\text { of grains }\end{array}$ & $5.5 \times 10^{9}$ & $\mathrm{~Pa}$ \\
\hline$\phi$ & Porosity of the porous medium & 0.345 & - \\
\hline$\rho_{s}$ & Solid density & $2.212 \times 10^{3}$ & $\mathrm{~kg} / \mathrm{m}^{3}$ \\
\hline$k_{0}$ & Permeability & $3.1 \times 10^{-12}$ & $\mathrm{~m}^{2}$ \\
\hline$\alpha_{\infty}$ & Tortuosity of the porous medium & 2.1 & - \\
\hline
\end{tabular}

${ }^{\mathrm{a}}$ Johnson and Plona (1982).

${ }^{b}$ Lide (2010).

${ }^{c}$ Pride (1994); Johnson et al. (1994); Jocker and Smeulders (2009). 
amplitude as the distance from the interface increases. The measured (true) peak-to-peak amplitude at $(r, z)=(0,-0.3) \mathrm{cm}$ is approximately $0.25 \mathrm{mV}$. In a separate experiment, acoustic pressure was measured along the $z$-axis. In Figure $2 b$, we show the acoustic waveforms measured with a Specialty Engineering Associates needle hydrophone (model PZT-Z44-1000-S/N), calibrated over the frequency range of interest. From these measurements, we can extrapolate that the acoustic pulse will indeed arrive at the interface around $0.1 \mathrm{~ms}$.

\section{FORWARD MODELING OF THE SEISMOELECTRIC INTERFACE RESPONSE}

The acoustic source is modeled by (e.g., Hall, 1987)

$$
\hat{p}(\omega, R, \theta)=\frac{A(\omega)}{R} e^{-i k R} D(\theta),
$$

where $\omega$ is the radial frequency, $R=\sqrt{\left(r-r_{0}\right)^{2}+\left(z-z_{0}\right)^{2}}$ is the distance to the source, $\theta$ is the angle of incidence (see Figure 3), $A(\omega)$ is the amplitude spectrum, and $k$ is the fluid wavenumber. The directivity function $D(\theta)$ is given by
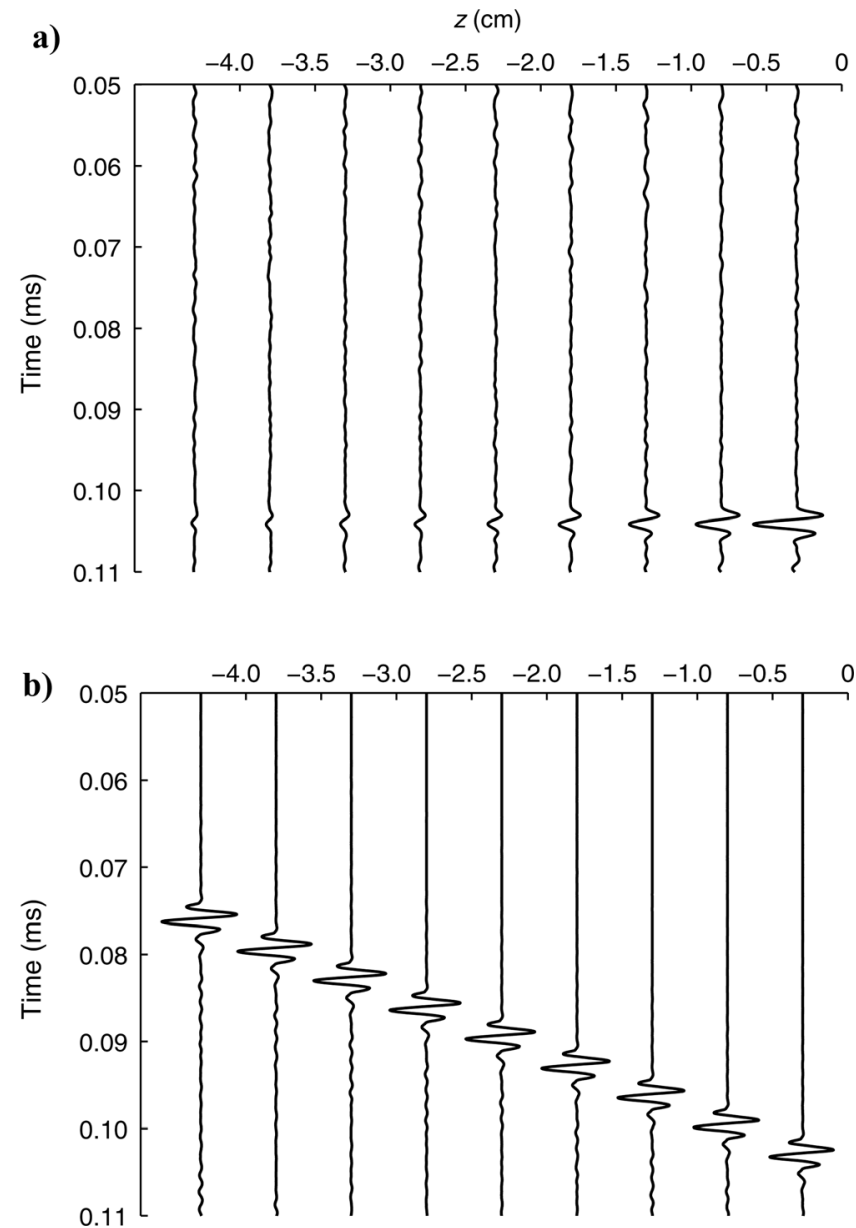

Figure 2. (a) Electric potential and (b) acoustic pressure as a function of vertical distance $z$ at $r=0$.

$$
D(\theta)=\frac{J_{1}(k a \sin \theta)}{k a \sin \theta} .
$$

Here, $J_{1}$ is the Bessel function of the first kind and first order, and $a$ is the radius of the transducer. At $(r, z)=(0,0), A(\omega)$ is determined directly from the measurement (see Figure 4$)$. As an additional test, the source response as a function of $r$ is measured for a $15.3 \mathrm{~cm}$ on-axis distance from the transducer. A single sine $500 \mathrm{kHz}$ source pulse with a $500 \mathrm{mV}$ peak-to-peak amplitude is used to excite the source transducer. Results are shown by the asterisks in Figure 5. The dashed line is the theoretical value obtained from equation 1 . We notice that the wavefield of the transducer is well described by equations 1 and 2 , although the theory predicts interference patterns that are not recorded in the experiment.

The modeled source pressure wavefield is now expanded into conical waves by means of the Sommerfeld integral (see e.g., Brekhovskikh [1960] and Aki and Richards [2002]). The total reflected electric potential $\hat{\varphi}\left(\omega, r_{r}, z_{r}\right)$ can then be expressed in terms of a Sommerfeld integral $\left(r_{0}=0 ; z_{0}, z_{r}<0\right)$ :

$$
\begin{aligned}
\hat{\varphi}\left(\omega, r_{r}, z_{r}\right)= & -i A(\omega) \int_{0}^{\infty} \frac{k_{r}}{k_{z}} D\left(k_{r}\right) J_{0}\left(k_{r} r_{r}\right) \\
& \times e^{i k_{z} z_{0}} e^{i k_{z}^{E} z_{r}} R^{E}\left(k_{r}\right) d k_{r},
\end{aligned}
$$

where $k_{r}=k \sin \theta$ and $k_{z}=k \cos \theta$ are the radial and vertical components of $k$, respectively, and $k_{z}^{E}$ is the vertical component of the fluid EM wavenumber. The seismoelectric reflection coefficient $R^{E}\left(k_{r}\right)$ relates the incident pressure wavefront to a reflected EM signal as derived by Schakel and Smeulders (2010), adopting full electrokinetic theory (Pride, 1994). Note that their coefficient is displacement normalized, whereas $R^{E}$ is pressure normalized (in $\mathrm{V} / \mathrm{Pa}$ ).

Expressing $k_{r}$ and $k_{z}$ in terms of $k$ and $\theta$, equation 3 is written as

$$
\begin{aligned}
\hat{\varphi}\left(\omega, r_{r}, z_{r}\right)= & -i A(\omega) \int_{0}^{\pi / 2+i \infty} D(\theta) k \sin \theta J_{0}\left(k r_{r} \sin \theta\right) \\
& \times e^{i k z_{0} \cos \theta} e^{i k k_{z}^{E}(\theta) z_{r}} R^{E}(\theta) d \theta
\end{aligned}
$$

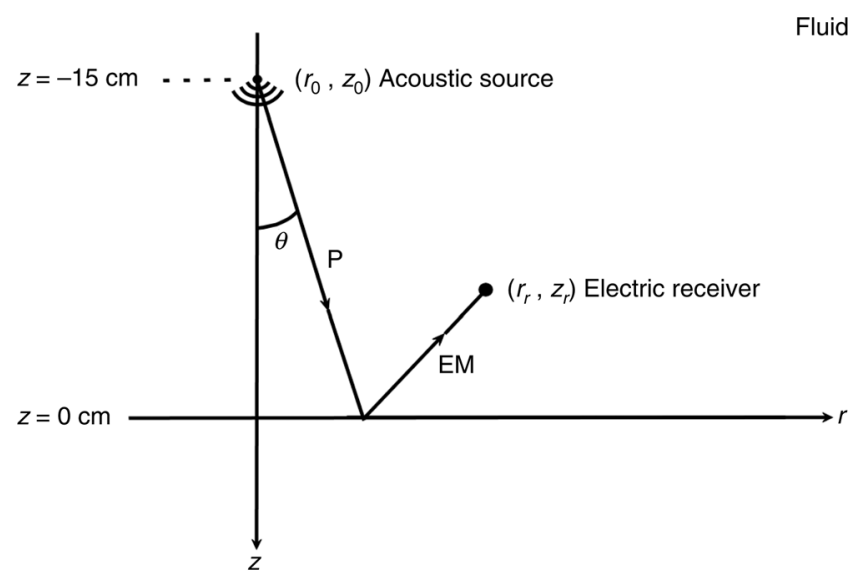

Porous medium

Figure 3. Geometry of seismoelectric model and experiment. A pressure (P-) wave is converted to an EM wave at the interface. 

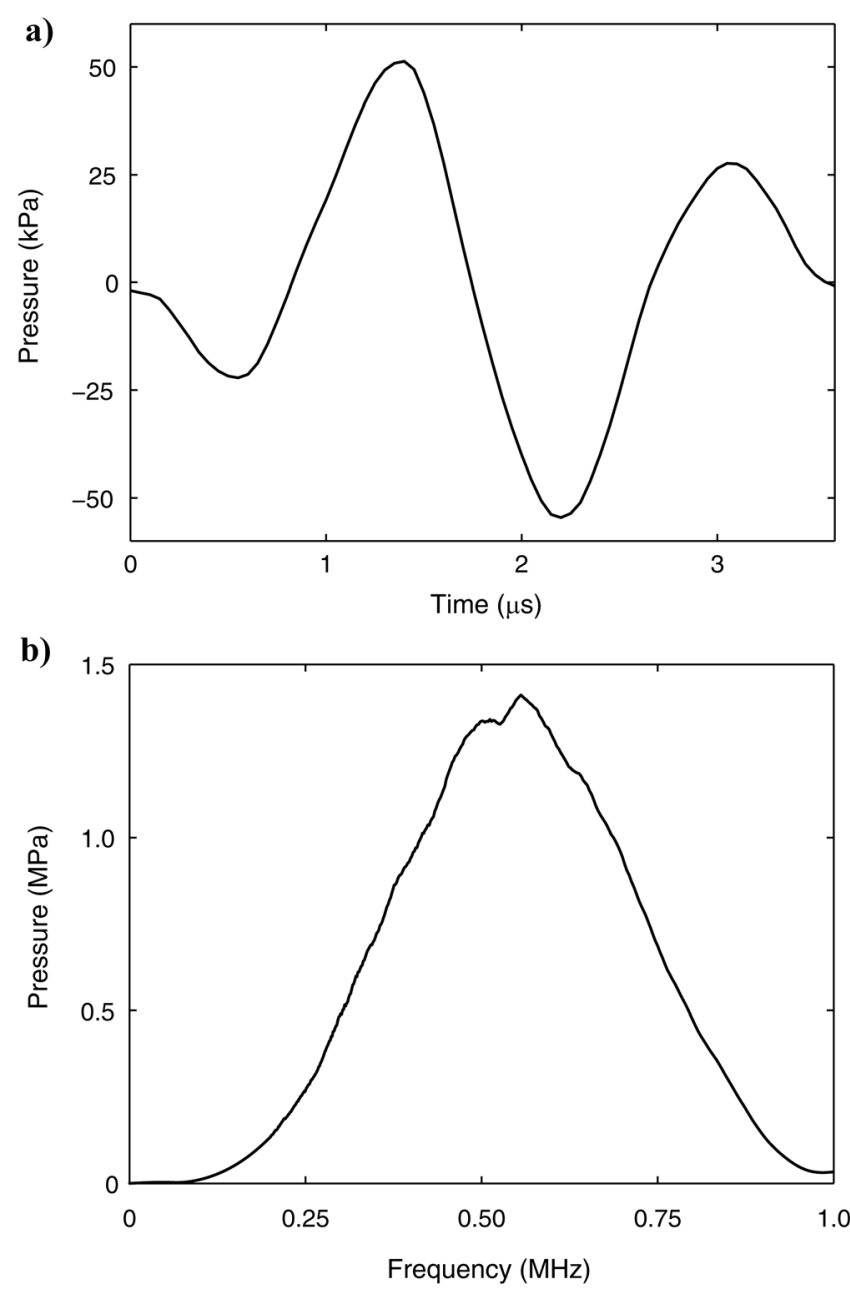

Figure 4. (a) Measured pressure pulse and (b) its frequency spectrum at $(r, z)=(0,0)$.

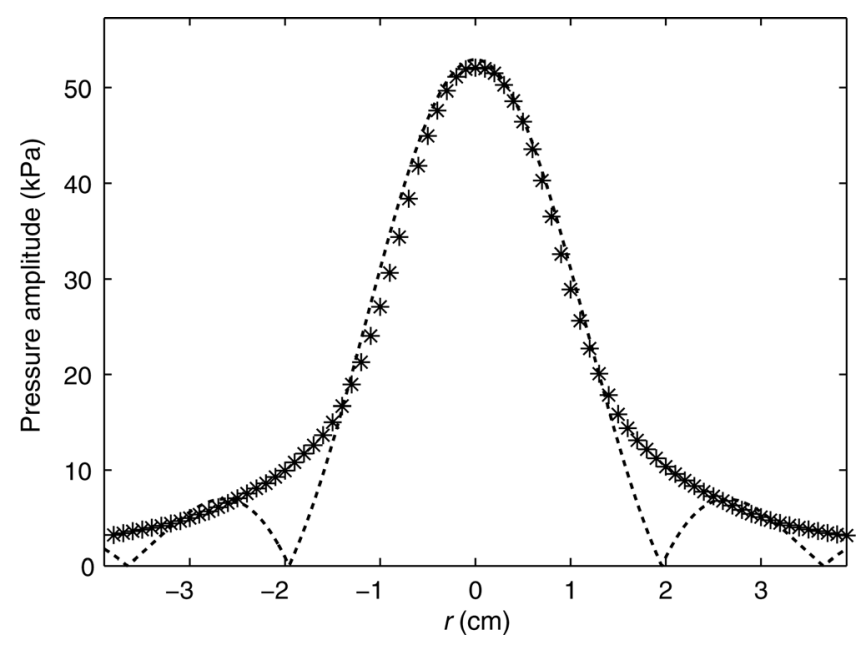

Figure 5. Measured (asterisks) and modeled (dashed line) pressure as a function of $r$. On-axis distance from the transducer is $15.3 \mathrm{~cm}(R=15.3 \mathrm{~cm}$ when $\theta=0)$. where $k_{z}^{E}(\theta)=\omega \sqrt{\left(1 / c_{E}^{2}(\omega)\right)-\left(\sin ^{2} \theta / c_{P}^{2}\right)}$ and $\operatorname{Im}\left[k_{z}^{E}(\theta)<0\right]$. Fluid EM and pressure wave velocities are given by, respectively, $\quad c_{E}(\omega)=\sqrt{\left(\mu \varepsilon_{0} \epsilon_{f}-\left(i \mu \sigma_{f l} / \omega\right)\right)^{-1}}$ and $\quad c_{P}=\sqrt{K_{f} / \rho_{f}}$, where $\varepsilon_{0}$ is the vacuum permittivity and $\sigma_{f l}$ is the fluid conductivity. The path of integration is along straight lines from zero to $\pi / 2$ and from $\pi / 2$ to $\pi / 2+i \infty$ in the complex $\theta$ plane.

The second integral over complex $\theta$ is simplified further using the substitution $\theta=\pi / 2+i \ln \left[\sqrt{\gamma^{2}+1}+\gamma\right]$ :

$$
\begin{aligned}
\hat{\varphi}\left(\omega, r_{r}, z_{r}\right)= & -\frac{i A(\omega)}{a} \int_{0}^{\pi / 2} J_{0}\left(k r_{r} \sin \theta\right) J_{1}(k a \sin \theta) \\
& \times e^{i k z_{0} \cos \theta} e^{i k_{z}^{E}(\theta) z_{r}} R^{E}(\theta) d \theta \\
& +\frac{A(\omega)}{a} \int_{0}^{\infty} J_{0}\left(k r_{r} \sqrt{\gamma^{2}+1}\right) \frac{J_{1}\left(k a \sqrt{\gamma^{2}+1}\right)}{\sqrt{\gamma^{2}+1}} \\
& \times e^{k z_{0} \gamma} e^{i k_{z}^{E}(\gamma) z_{r}} R^{E}(\gamma) d \gamma
\end{aligned}
$$

where equation 2 is used for $D(\theta)$. Equation 5 can be readily evaluated numerically and yields the total reflected EM wavefield through the inverse fast Fourier transform. The source is located at $\left(r_{0}, z_{0}\right)=(0,-15) \mathrm{cm}$ (Figure 3$)$. For each frequency, we use a recursive adaptive Simpson quadrature algorithm implemented in Matlab. A $144-896 \mathrm{kHz}$ numerical band-pass filter is applied, and the input parameters of Tables 1 and 2 are used. Figure 6 shows the resulting modeled reflected electric potential and its frequency spectrum for a $10^{-3} \mathrm{M} \mathrm{NaCl}$ salinity.

\section{COMPARISON BETWEEN EXPERIMENT AND FORWARD MODEL}

Figure 7 compares measured waveforms (solid lines) at various positions in the fluid along the negative $z$-axis (Figure 3 ) with modeled waveforms (dashed lines). The agreement between measured and modeled waveforms is excellent. The modeled amplitudes are scaled to the measured amplitudes by the amplitude field scaling factor $A_{C}=0.19$, which is obtained by comparing the theoretical Fourier spectrum maxima of the entire field to the measured maxima. Thus, $A_{C}<1$ means that the theory overpredicts the actual measured electric potentials.

In Figure 8, the scaled theoretical Fourier spectra are compared with the experiments. Note that the measured pulses have

Table 2. Electrochemical parameters and amplitude field scaling factors $A_{C}$. Conductivities were measured directly in the fluid surrounding the porous medium, whereas zeta potentials $\zeta$ were obtained from Zetasizer Nano ZS measurements on crushed porous sample material suspensions in the corresponding salt solutions.

\begin{tabular}{lccc} 
Salinity $(\mathrm{M} \mathrm{NaCl})$ & Conductivity $(\mathrm{S} / \mathrm{m})$ & $\zeta(\mathrm{mV})$ & $A_{C}$ \\
\hline $10^{-4}$ & $1.27 \times 10^{-3}$ & -51.7 & 0.03 \\
$10^{-3}$ & $1.20 \times 10^{-2}$ & -61.5 & 0.19 \\
$10^{-2}$ & $1.01 \times 10^{-1}$ & -58.1 & 0.41 \\
\hline
\end{tabular}


their energy distributed over slightly smaller frequencies than predicted by theory. Figure 9 compares measured waveforms (solid lines) at various positions in the fluid along the $r$-axis at $z=-1.3 \mathrm{~cm}$ (Figure 3 ) with modeled waveforms (dashed lines). Again, $A_{C}=0.19$ is used. As in the measurements along the $z$ axis, good agreement in the waveforms as well as in the spatial amplitude pattern is observed. We notice a slight asymmetry in the measured data. This is most likely caused by a small misalignment in the setup. In Figure 10, the corresponding fre-
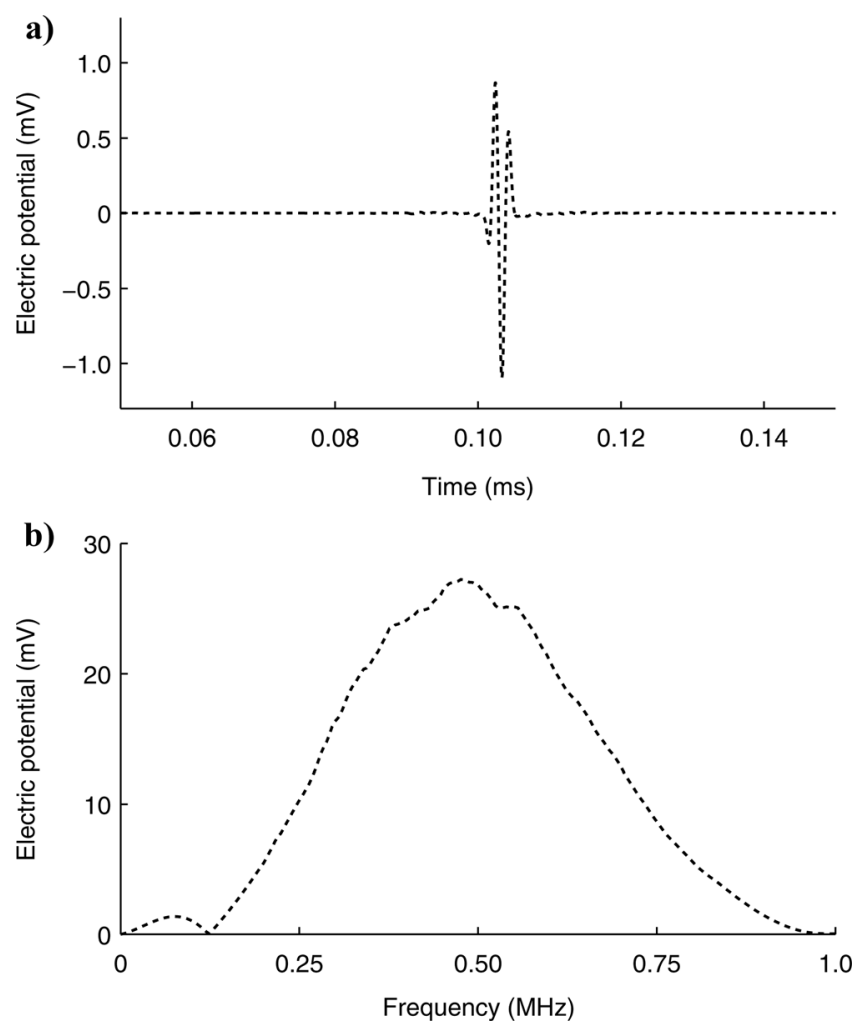

Figure 6. (a) Modeled reflected electric potential for an acoustic source at $\left(r_{0}, z_{0}\right)=(0,-15) \mathrm{cm}$ and $(\mathrm{b})$ its frequency spectrum.

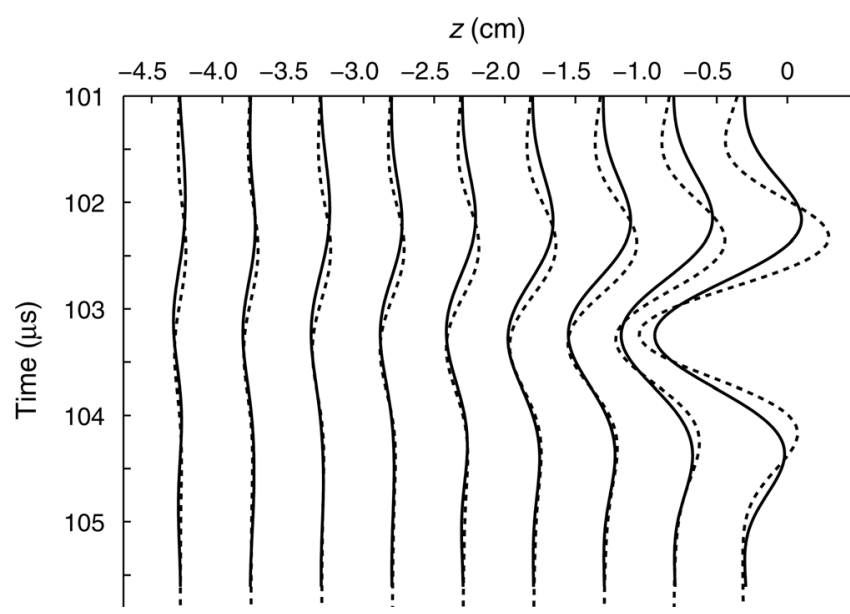

Figure 7. Measured (solid lines) and modeled (dashed lines) electric potential waveforms at various positions in the fluid along the $z$-axis at $r=0$ for $C=10^{-3} \mathrm{M} \mathrm{NaCl}$. quency spectra are shown. We recall that the $A_{C}=0.19$ value is obtained from Fourier spectra of the entire field shown in Figures 8 and 10 .

To compare predicted amplitudes with measurements more directly, peak-to-peak amplitudes $\left(V_{p p}\right)$ are plotted in Figure 11 and compared with our model (dashed lines). Again, the spatial amplitude pattern is excellently predicted by theory for the measurements along the negative $z$-axis (Figure 11a) and parallel to the $r$-axis at $z=-1.3 \mathrm{~cm}$ (Figure 11b). We also check the repeatability of the experiment. There is a small amplitude decrease over two days. The waveform is preserved on these longer timescales. In Figure 11 (gray lines), we also plot the vertical electric dipole approximation $A_{D} z e^{-i k^{E} d} /\left(r^{2}+z^{2}\right)^{3 / 2}$ (see also, e.g., Dupuis et al. [2007] and Haines et al. [2007]), where $d$ is the distance from the origin and the coefficient $A_{D}$ is

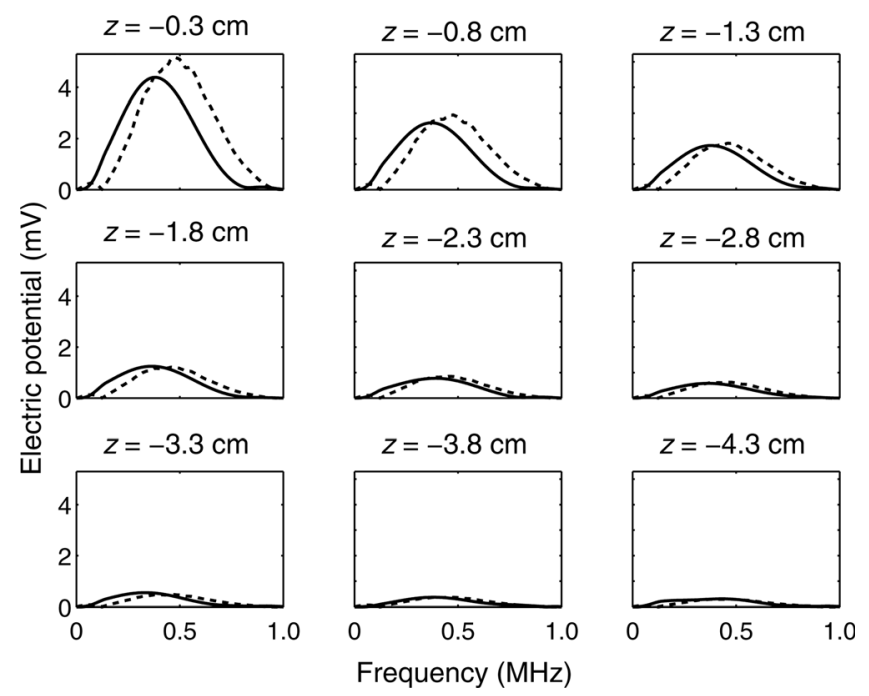

Figure 8. Measured (solid lines) and modeled (dashed lines) electric potential spectra at various positions in the fluid along the $z$-axis at $r=0$ for $C=10^{-3} \mathrm{M} \mathrm{NaCl}$.

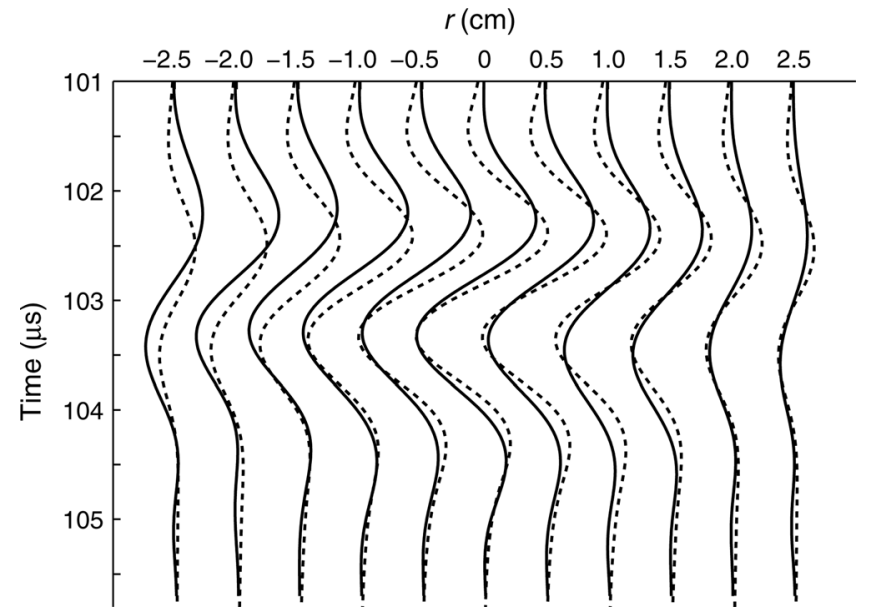

Figure 9. Measured (solid lines) and modeled (dashed lines) electric potential waveforms at various positions in the fluid parallel to the $r$-axis at $z=-1.3 \mathrm{~cm}$ for $C=10^{-3} \mathrm{M} \mathrm{NaCl}$. 
obtained from a best-fit procedure. The vertical electric dipole approximation overestimates the spatial amplitude decay. This means that the full model (equation 5) is needed to explain the amplitude measurements.
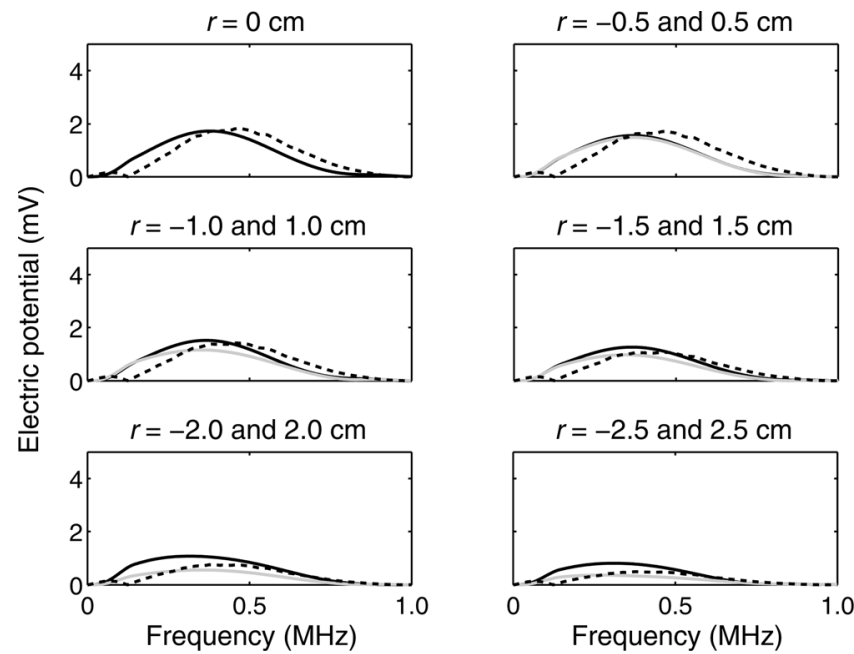

Figure 10. Measured (solid lines) and modeled (dashed lines) electric potential spectra at various positions in the fluid parallel to the $r$-axis at $z=-1.3 \mathrm{~cm}$ for $C=10^{-3} \mathrm{M} \mathrm{NaCl}$. The black and gray solid lines correspond with measured spectra for negative and positive $r$-coordinates, respectively.
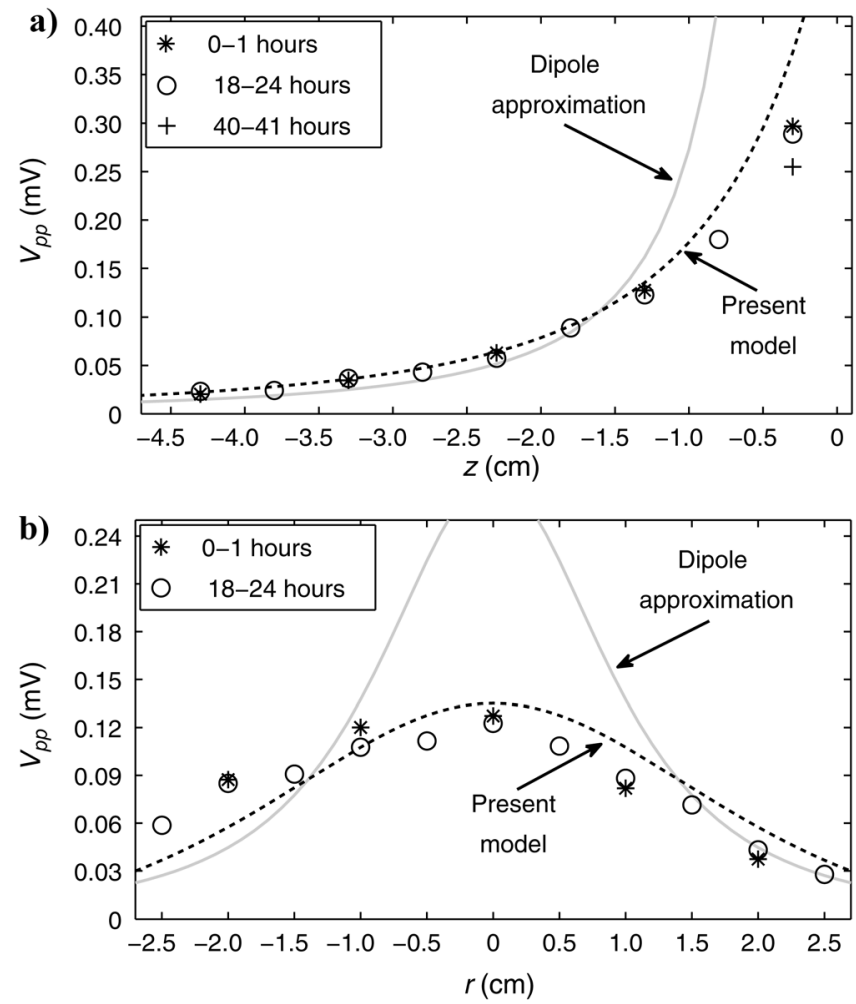

Figure 11. Measured (symbols) and modeled (dashed lines) peakto-peak amplitudes $V_{p p}$ as a function of (a) $z$ at $r=0$ and (b) $r$ at $z=-1.3 \mathrm{~cm}$ for $C=10^{-3} \mathrm{M} \mathrm{NaCl}$. Experiments were repeated several times to check repeatability. Here, $A_{C}=0.19$. Also, the electric dipole approximation is plotted (gray lines).
Figures 12 and 13 show measured and full-waveform modeled $V_{p p}$-values where $C=10^{-4}$ and $C=10^{-2} \mathrm{M} \mathrm{NaCl}$ solutions are used, respectively. We determine the $A_{C}$-values in the same way as for the $10^{-3} \mathrm{M} \mathrm{NaCl}$ solution. Resulting values are given in Table 2. The spatial amplitude pattern is also excellently predicted for these salt solutions.

For higher salinities, the $A_{C}$ values are closer to (or approach) unity. This observation is in agreement with Block and Harris (2006), who find that the discrepancy between electrokinetic theoretical predictions and measurements decreases with increasing salinity. Block and Harris (2006) also suggest that when low-salinity electrolytes are used to saturate the pores of sandstones, surface conductivity effects other than those predicted by Pride's electrokinetic theory may become dominant. The Pride theory used here incorporates diffuse-layer excess conduction associated with electromigration and electroosmosis. When the pore width is much larger than the Debye length, which is a measure of the diffuse-layer thickness, surface conductivity effects become negligible with increasing salinity. Our sample has a $16-40 \mu \mathrm{m}$ pore width, whereas the Debye length is on the order of 3-30 $\mathrm{nm}$.

We also observe that for all salinities, the $A_{C}$ values differ from unity. Several possible reasons can be identified. First, the zeta potentials are obtained from crushed sample material suspensions. A Zetasizer is used for this. Probably, the zeta potential measured on ground-up material has a much higher value than that measured on intact (aged) surface material. This observation alone could be responsible for $A_{C}$ being different from
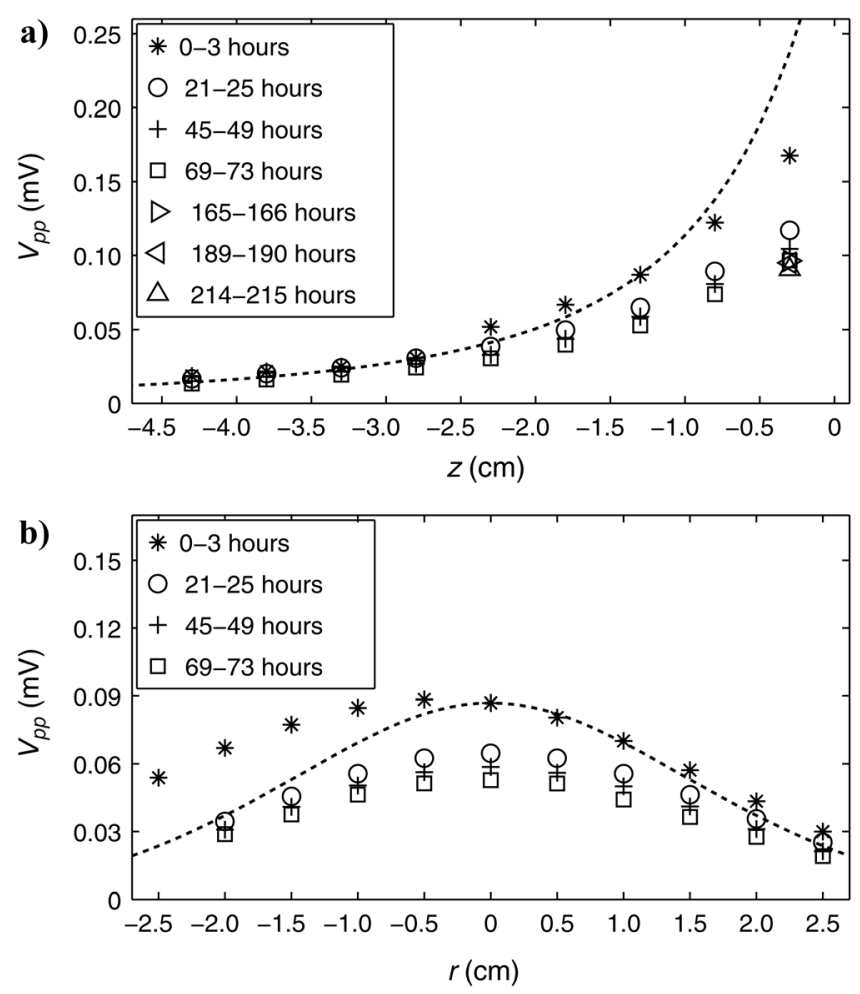

Figure 12. Measured (symbols) and modeled (dashed lines) peakto-peak amplitudes $V_{p p}$ as a function of (a) $z$ at $r=0$ and (b) $r$ at $z=-1.3 \mathrm{~cm}$ for $C=10^{-4} \mathrm{M} \mathrm{NaCl}$. Experiments were repeated several times to check repeatability. Here, $A_{C}=0.03$. 

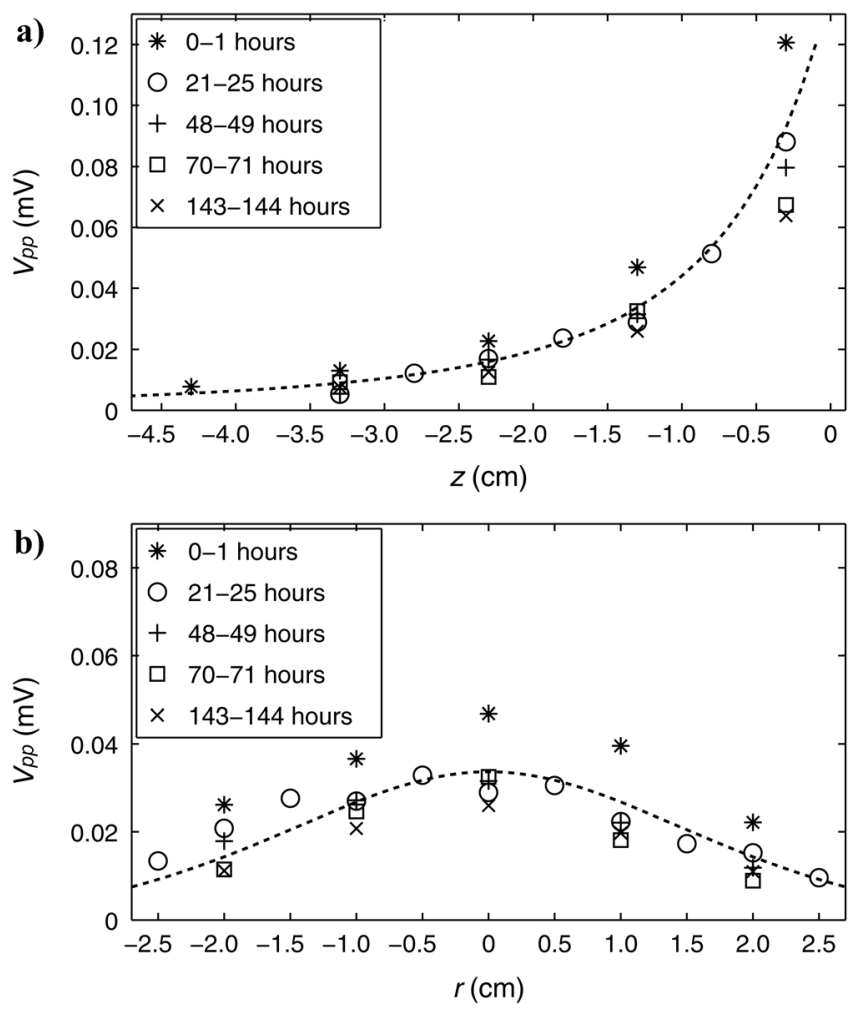

Figure 13. Measured (symbols) and modeled (dashed lines) peakto-peak amplitudes $V_{p p}$ as a function of (a) $z$ at $r=0$ and (b) $r$ at $z=-1.3 \mathrm{~cm}$ for $C=10^{-2} \mathrm{M} \mathrm{NaCl}$. Experiments were repeated several times to check repeatability. Here, $A_{C}=0.41$.

unity. We also performed zeta potential calibration measurements on $99.8 \%$ silicon oxide $\left(\mathrm{SiO}_{2}\right)$ powder suspensions. The measured values were different by a factor of approximately two to three from streaming potential measurements reported in literature (e.g., Li and de Bruyn [1966] and Pride and Morgan [1991]), which indicates the uncertainty in our zeta potential determination. Second, the fluid conductivities given in Table 2 are measured in the fluid outside the pores; therefore, the actual pore fluid conductivity may be different. Finally, the seismoelectric interface response amplitudes decrease on timescales that are longer (approximately 24 hours) than the duration of a single measurement series (1-4 hours). For example, the measured amplitudes in Figure 13 from the last measurement series (143144 hours) are, on average, 1.9 times smaller than those of the first series (0-1 hours). This observation could be related to increasing bulk conductivity (Block and Harris, 2006) and/or incomplete equilibration of the electric double layer. For silica, the electric double layer may take several days to become stable (Morgan et al., 1989), and it may take hours at soil interfaces (Liu et al., 2008). One could also speculate on the electrodes developing a voltage drop at their surface, thus reducing the measured amplitudes over time. However, replacing the electrodes under stable experimental conditions does not change potential readings significantly.

\section{CONCLUSIONS}

The electrokinetic conversion of an acoustic wave to an EM wave at an interface was systematically investigated in a labora- tory setup. We independently determined the mechanical and electrical parameters. It was demonstrated that this effect is well predicted by electrokinetic theory in terms of waveform and spatial amplitude pattern. At $C=10^{-3}$ and $C=10^{-2} \mathrm{M} \mathrm{NaCl}$, amplitude field scaling factors of 0.19 and 0.41 were used. At $C=10^{-4} \mathrm{M} \mathrm{NaCl}$, however, the value was only 0.03 , which may be related to surface conductivity effects other than those present in electrokinetic theory. The magnitude of acoustic-toEM conversion was shown to depend on the salinity of the saturating fluid, which is promising for applications in the oil and gas industry because the zones of interest are saturated with (resistive) hydrocarbons instead of water. A careful characterization of the source radiation pattern was part of the forward model, indicating that electrokinetic interface response predictions require proper modeling of the source. The results of this study indicate that the acoustic-to-EM wave responses of these rock/fluid combinations can be predicted by a full-waveform model for general subsurface exploration purposes.

\section{ACKNOWLEDGMENTS}

This work was performed under the financial support of ShellFOM within the Physics of Fluids and Sound Propagation research program. The authors are grateful to Steven Huynink and Krishna Kowlgi of the Delft University Applied Sciences department for measuring zeta potentials and also to Caroline van der Lee for determining elastic bulk moduli. The technical support of Jolanda van Haagen is greatly appreciated. We thank the anonymous reviewers for valuable comments. M. Schakel also thanks Zhenya Zhu, Gabriel Chao, Bobby Hak, Christiaan Schoemaker, and Karel van Dalen for valuable discussions.

\section{REFERENCES}

Aki, K., and P. G. Richards, 2002, Quantitative seismology, 2nd ed.: University Science Books.

Beamish, D., 1999, Characteristics of near-surface electrokinetic coupling: Geophysical Journal International, 137, no. 1, 231-242, doi:10.1046/j.1365-246x.1999.00785.x.

Block, G. I., and J. G. Harris, 2006, Conductivity dependence of seismoelectric wave phenomena in fluid-saturated sediments: Journal of Geophysical Research, 111, B01304, doi:10.1029/2005JB003798.

Bordes, C., L. Jouniaux, M. Dietrich, J.-P. Pozzi, and S. Garambois, 2006, First laboratory measurements of seismo-magnetic conversions in fluidfilled Fontainebleau sand: Geophysical Research Letters, 33, no. 1, L01302, doi:10.1029/2005GL024582.

Bordes, C., L. Jouniaux, S. Garambois, M. Dietrich, J.-P. Pozzi, and S. Gaffet, 2008, Evidence of the theoretically predicted seismo-magnetic conversion: Geophysical Journal International, 174, 489-504, doi:10.1111/j.1365-246X.2008.03828.x.

Brekhovskikh, L. M., 1960, Waves in layered media: Academic Press Inc.

Butler, K. E., R. D. Russell, A. W. Kepic, and M. Maxwell, 1996, Measurement of the seismoelectric response from a shallow boundary: Geophysics, 61, 1769-1778, doi:10.1190/1.1444093.

Charara, M., I. Zaretsky, and M. Zamora, 2009, Seismoelectric modeling of laboratory experiments: 71st Annual International Conference \& Technical Exhibition, EAGE, Extended Abstracts, Z006.

Dupuis, J. C., and K. E. Butler, 2006, Vertical seismoelectric profiling in a borehole penetrating glaciofluvial sediments: Geophysical Research Letters, 33, L16301, doi:10.1029/2006GL026385.

Dupuis, J. C., K. E. Butler, and A. W. Kepic, 2007, Seismoelectric imaging of the vadose zone of a sand aquifer: Geophysics, 72, no. 6, A81A85, doi:10.1190/1.2773780.

Garambois, S., and M. Dietrich, 2001, Seismoelectric wave conversions in porous media: Field measurements and transfer function analysis: Geophysics, 66, 1417-1430, doi:10.1190/1.1487087. 
2002, Full waveform numerical simulations of seismoelectromagnetic wave conversions in fluid-saturated stratified porous media: Journal of Geophysical Research, 107, 2148, doi:10.1029/2001JB000316.

Haartsen, M. W., and S. R. Pride, 1997, Electroseismic waves from point sources in layered media: Journal of Geophysical Research, 102, 24745-24769, doi:10.1029/97JB02936.

Haines, S. S., and S. R. Pride, 2006, Seismoelectric numerical modeling on a grid: Geophysics, 71, no. 6, N57-N65, doi:10.1190/1.2357789.

Haines, S. S., S. R. Pride, S. L. Klemperer, and B. Biondi, 2007, Seismoelectric imaging of shallow targets: Geophysics, 72, no. 2, G9-G20, doi:10.1190/1.2428267.

Hall, D. E., 1987, Basic acoustics: John Wiley \& Sons, Inc.

Jocker, J., and D. Smeulders, 2009, Ultrasonic measurements on poroelastic slabs: Determination of reflection and transmission coefficients and processing for Biot input parameters: Ultrasonics, 49, no. 3, 319-330, doi:10.1016/j.ultras.2008.10.006.

Johnson, D. L., D. L. Hemmick, and H. Kojima, 1994, Probing porous media with first and second sound. I. Dynamic permeability: Journal of Applied Physics, 76, no. 1, 104-114, doi:10.1063/1.357116.

Johnson, D. L., and T. J. Plona, 1982, Acoustic slow waves and the consolidation transition: Journal of the Acoustical Society of America, 72 556-565, doi:10.1121/1.388036.

Li, H. C., and P. L. de Bruyn, 1966, Electrokinetic and adsorption studies on quartz: Surface Science, 5, no. 2, 203-220, doi:10.1016/00396028(66)90082-3.

Lide, D. R., ed., 2010, CRC handbook of chemistry and physics, 90th ed. (Internet): CRC Press/Taylor and Francis.

Liu, Z., L. Yuan, X. Zhang, Z. Liu, and H. Wu, 2008, A laboratory seismoelectric measurement for the permafrost model with a frozen-unfrozen interface: Geophysical Research Letters, 35, L21404, doi:10.1029/ 2008GL035724.

Long, L. T., and W. K. Rivers, 1975, Field measurement of the electroseismic response: Geophysics, 40, 233-245, doi:10.1190/1.1440521.
Mikhailov, O. V., M. W. Haartsen, and M. N. Toksöz, 1997, Electroseismic investigation of the shallow subsurface: Field measurements and numerical modeling: Geophysics, 62, 97-105, doi:10.1190/ 1.1444150

Morgan, F. D., E. R. Williams, and T. R. Madden, 1989, Streaming potential properties of Westerly granite with applications: Journal of Geophysical Research, 94, 12449-12461, doi:10.1029/JB094iB09p12449.

Pride, S., 1994, Governing equations for the coupled electromagnetics and acoustics of porous media: Physical Review B: Condensed Matter and Materials Physics, 50, 15678-15696, doi:10.1103/PhysRevB.50.15678.

Pride, S. R., and M. W. Haartsen, 1996, Electroseismic wave properties: Journal of the Acoustical Society of America, 100, 1301-1315, doi:10.1121/1.416018.

Pride, S. R., and F. D. Morgan, 1991, Electrokinetic dissipation induced by seismic waves: Geophysics, 56, 914-925, doi:10.1190/1.1443125.

Reppert, P. M., F. D. Morgan, D. P. Lesmes, and L. Jouniaux, 2001, Frequency-dependent streaming potentials: Journal of Colloid and Interface Science, 234, no. 1, 194-203, doi:10.1006/jcis.2000.7294.

Schakel, M., and D. Smeulders, 2010, Seismoelectric reflection and transmission at a fluid/porous-medium interface: Journal of the Acoustical Society of America, 127, no. 1, 13-21, doi:10.1121/1.3263613.

Zhu, Z., M. W. Haartsen, and M. N. Toksöz, 1999, Experimental studies of electrokinetic conversions in fluid-saturated borehole models: Geophysics, 64, 1349-1356, doi:10.1190/1.1444639.

, 2000 , Experimental studies of seismoelectric conversions in fluidsaturated porous media: Journal of Geophysical Research, 105, no. B12, 28055-28064, doi:10.1029/2000JB900341.

Zhu, Z., and M. N. Toksöz, 2003, Crosshole seismoelectric measurements in borehole models with fractures: Geophysics, 68, 1519-1524, doi:10.1190/1.1620625.

2005, Seismoelectric and seismomagnetic measurements in fractured borehole models: Geophysics, 70, no. 4, F45-F51, doi:10.1190/ 1.1996907. 